\title{
Matching Conditional Moments in PDF modelling of non-premixed combustion
}

\author{
A.Y.Klimenko \\ Mech. Eng. Division, The University of Queensland Qld 4072, Australia \\ Email:klimenko@mech.uq.edu.au
}

\begin{abstract}
The rate of generation of fluctuations with respect to the scalar values conditioned on the mixture fraction, which significantly affects turbulent non-premixed combustion processes, is examined in the paper. Simulation of the rate in major mixing model is investigated and the derived equations can assist in selecting the model parameters so that level of conditional fluctuations is better reproduced by the models. A more general formulation of the Multiple Mapping Conditioning (MMC) model that distinguishes the reference and conditioning variables is suggested. This formulation can be viewed as methodology of enforcing certain desired conditional properties onto conventional mixing models. Examples of constructing consistent MMC models with dissipation and velocity conditioning as well as of combining MMC with Large Eddy Simulations (LES) are also provided in the paper.
\end{abstract}

Key words: turbulent non-premixed combustion modelling PACS: 82.33.Vx, 47.27.Eq

\section{Introduction}

The models of nonpremixed turbulent combustion are traditionally divided into two categories the mixture fraction-based models (fast chemistry [1] and Flamelet [2]) and the Probability Density Function (PDF) models[3,4]. The appearance of Conditional Moment Closure (CMC, [5]) seems to blur this division since CMC has links with the both categories. The CMC model has been developed independently by and in cooperation between R.W. Bilger and the author of this work. Originally, the CMC model, which uses the mixture fraction as an independent variable and, at the same time, allows for consistent specification of the global convective scalar transport, was designed as a specific combustion model introducing a remedy for inherent deficiencies of the

Published: Combustion and Flame, 143(4), pp. 369-385, 2005 
Flamelet models. At present CMC, as a methodology of dealing with conditional expectations, has a much broader role in studies of turbulence. The approach pursued in the present work indicates that CMC is linked to the PDF methods as much as it is related to the mixture fraction-based models. R.W. Bilger, who actually suggested the title of the model that outlined the importance of the conditional moments, must have foreseen this broader role of CMC.

Section 1 introduces basic CMC equations and provides analysis of the generation rate for conditional fluctuations based on the Kolmogorov theory of turbulence. The present work examines further the effect of turbulent mixing that increases the dimension of the accessed domain in the composition space that was analysed by Pope [6] and introduces a quantitative measure for this effect that is expressed in terms of the conditional variance generation.

The PDF methods are most accurate in specification of the reaction source terms but turbulent mixing is replaced in these models by a surrogate mixing process. While the average properties are directly enforced on surrogate mixing by setting the required rate of the average dissipation, matching the conditional properties (such as the rate of generation of conditional fluctuations) would be a pure coincidence. In Section 2, we estimate the rate of generation of conditional fluctuations for surrogate mixing in most common mixing models. With the use of these equations, one can achieve a better match with the expected physical level of the conditional variance even within the limits of conventional mixing modelling. The Multiple Mapping Conditioning (MMC, $[7,8])$ can be viewed as a methodology of enforcing certain conditional properties on surrogate mixing by mapping the reactive scalars into the reference space (MMC effectively generalises the Mapping Closure concept [9-11]). Thus, the significance of conditional characteristics in MMC modelling is even more profound. Since the basic MMC is, generally, consistent with the 1st order CMC equation [7], the constraints derived from consideration of the conditional variances are of prime interest.

While conditioning on the scalar values have been studied within the CMC framework for some time, we know much less about the other possible types of conditioning (ref.[12], where joint scalar/dissipation conditioning is considered, represents a notable exception). Section 4 introduces a most general understanding of MMC that allows to directly emulate the properties of turbulence which appear to be most important for combustion simulation. MMC restricts the influence of the surrogate mixing to a locality defined with the use of the selected properties and physical coordinates. Section 5 introduces examples of MMC models in which the additional characteristics of turbulence - scalar dissipation and velocities - are directly emulated. 


\section{Moments conditioned on the mixture fraction}

\subsection{The CMC Equations}

In high Reynold number flows, the joint Favre PDF $P_{Y}=P_{Y}\left(Y^{(0)}, \ldots, Y^{(n)} ; \mathbf{x}, t\right)$ of the scalars $Y^{(0)}, \ldots, Y^{(n)}$ that are governed by equations

$$
\rho \frac{\partial Y^{(i)}}{\partial t}+\rho \mathbf{u} \cdot \nabla Y^{(i)}-\nabla \cdot\left(\rho D \nabla Y^{(i)}\right)=W^{(i)}
$$

satisfies the following equation

$$
\frac{\partial \overline{\boldsymbol{\rho}} P_{Y}}{\partial t}+\boldsymbol{\nabla} \cdot\left(\mathbf{u}_{Y} \overline{\boldsymbol{\rho}} P_{Y}\right)+\frac{\partial W_{Y}^{(i)} \overline{\boldsymbol{\rho}} P_{Y}}{\partial Y_{i}}+\frac{\partial^{2} N_{Y}^{(i j)} \overline{\boldsymbol{\rho}} P_{Y}}{\partial Y_{i} \partial Y_{j}}=0
$$

where

$$
\begin{aligned}
& N_{Y}^{(i j)} \equiv\left\langle N^{(i j)} \mid \mathbf{Y}\right\rangle, N^{(i j)} \equiv D \nabla Y^{(i)} \cdot \nabla Y^{(j)}, \quad \mathbf{u}_{Y} \equiv\langle\mathbf{u} \mid \mathbf{Y}\rangle \\
& W_{Y}^{(i)} \equiv\left\langle W^{(i)} \mid \mathbf{Y}\right\rangle, \quad \bar{\rho} \equiv\langle\rho\rangle
\end{aligned}
$$

and indices $i$ and $j$ run over all species considered in the PDF equations. A PDF model is consistent with the PDF equation if it implies a reasonable approximation for the coefficients $\mathbf{u}_{Y}$ and $N_{Y}^{(i j)}$ and with these coefficients the simulated PDF $P_{Y}$ satisfies equation (2). By default, The PDFs used in the present work are the Favre PDFs, for example, $\bar{\rho} P_{Y}=\rho_{Y} P_{Y}^{\circ}$ where $\rho_{Y} \equiv\langle\rho \mid \mathbf{Y}\rangle$ and $P_{Y}^{\circ}$ is the conventional PDF. Here and further in the paper we neglect the fluctuations of the density with respect to the conditional means. If these fluctuations are to be taken into account then the conditional averaging $\langle(\cdot) \mid \mathbf{Y}\rangle$ is to be replaced by the conditional Favre averaging $\langle(\cdot) \rho \mid \mathbf{Y}\rangle / \rho_{Y}$.

The CMC equations specify turbulent transport of scalars with respect to another scalar (or scalars), whose typical selection is represented by the mixture fraction $Z$. Assuming that $Z \equiv Y^{(0)}$ is the mixture fraction, we put $W^{(0)}=0$. The CMC equations are the governing equations for the conditional expectation $Q^{(i)} \equiv\left\langle Y^{(i)} \mid Z\right\rangle$ and the conditional variance $\Theta^{(i j)} \equiv\left\langle\theta^{(i j)} \mid Z\right\rangle, \theta^{(i j)} \equiv$ $\left(Y^{(i)}\right)^{\prime}\left(Y^{(j)}\right)^{\prime},\left(Y^{(i)}\right)^{\prime} \equiv Y^{(i)}-Q^{(i)}$. These equations can be obtained form the transport equations (1) (the decomposition technique) or directly from the PDF equation (2) (the PDF technique). The 1st and 2nd order unclosed CMC equations, which neglect only the terms that asymptotically small in the flows with high Reynolds numbers, take the form 


$$
\begin{gathered}
\frac{\partial Q^{(i)} \overline{\boldsymbol{\rho}} P_{Z}}{\partial t}+\boldsymbol{\nabla} \cdot\left(\left\langle\mathbf{u} Y^{(i)} \mid Z\right\rangle \overline{\boldsymbol{\rho}} P_{Z}\right)=\frac{\partial}{\partial Z}\left[N_{Z} \overline{\boldsymbol{\rho}} P_{Z} \frac{\partial Q^{(i)}}{\partial Z}-Q^{(i)} \frac{\partial N_{Z} \overline{\boldsymbol{\rho}} P_{Z}}{\partial Z}+J_{Y^{(i)}}\right]+W_{Z}^{(i)} \overline{\boldsymbol{\rho}} P_{Z} \\
\frac{\partial \Theta^{(i j)} \overline{\boldsymbol{\rho}} P_{Z}}{\partial t}+\boldsymbol{\nabla} \cdot\left(\left\langle\mathbf{u} \theta^{(i j)} \mid Z\right\rangle \overline{\boldsymbol{\rho}} P_{Z}\right)=\frac{\partial}{\partial Z}\left[N_{Z} \overline{\boldsymbol{\rho}} P_{Z} \frac{\partial \Theta^{(i j)}}{\partial Z}-\Theta^{(i j)} \frac{\partial N_{Z} \overline{\boldsymbol{\rho}} P_{Z}}{\partial Z}+J_{\theta^{(i j)}}\right]+ \\
\quad+\overline{\boldsymbol{\rho}} P_{Z}\left[\Psi_{N}^{(i j)}+\Psi_{N}^{(j i)}+\Psi_{V}^{(i j)}+\Psi_{V}^{(j i)}+\Psi_{W}^{(i j)}+\Psi_{W}^{(j i)}-\Psi_{D}^{(i j)}-\Psi_{D}^{(j i)}\right]
\end{gathered}
$$

where

$$
\begin{gathered}
J_{(\cdot)} \equiv 2\left\langle D \nabla(\cdot)^{\prime} \cdot \nabla Z \mid Z\right\rangle \overline{\boldsymbol{\rho}} P_{Z}-\frac{\partial\left\langle N^{\prime}(\cdot)^{\prime} \mid Z\right\rangle \overline{\boldsymbol{\rho}} P_{Z}}{\partial Z}, \\
\Psi_{N}^{(i j)} \equiv\left\langle N^{\prime}\left(Y^{(i)}\right)^{\prime} \mid Z\right\rangle \frac{\partial^{2} Q^{(j)}}{\partial Z^{2}}, \quad \Psi_{D}^{(i j)} \equiv\left\langle D \boldsymbol{\nabla}\left(Y^{(i)}\right)^{\prime} \cdot \nabla\left(Y^{(j)}\right)^{\prime} \mid Z\right\rangle, \\
\Psi_{V}^{(i j)} \equiv-\left\langle\mathbf{u}^{\prime}\left(Y^{(i)}\right)^{\prime} \mid Z\right\rangle \cdot \nabla Q^{(j)}, \quad \Psi_{W}^{(i j)} \equiv\left\langle\left(W^{(i)}\right)^{\prime}\left(Y^{(j)}\right)^{\prime} \mid Z\right\rangle, \\
N \equiv D(\boldsymbol{\nabla} Z)^{2}=N^{(00)},(\cdot)_{Z} \equiv\langle\cdot \mid Z\rangle,(\cdot)^{\prime} \equiv(\cdot)-\langle\cdot \mid Z\rangle
\end{gathered}
$$

and the Favre PDF of the mixture fraction, $P_{Z}$, is governed by

$$
\frac{\partial \overline{\boldsymbol{\rho}} P_{Z}}{\partial t}+\nabla \cdot\left(\langle\mathbf{u} \mid Z\rangle \overline{\boldsymbol{\rho}} P_{Z}\right)=-\frac{\partial^{2} N_{Z} \overline{\boldsymbol{\rho}} P_{Z}}{\partial Z^{2}}
$$

Most of the conditional moment equations, their derivation and closures are presented in Ref.[5] although the complete form of the PDF-derived unclosed conditional variance equation is given only in Kim[13] (one can note that the scalar transport equation (1) is used in [13] at several instances but it can be shown that all needed equations can be obtained by evaluating the moments of the joint PDF equation (2)). The equations are given here in divergent form, the alternative (but equivalent) convective form can be obtained using the mixture fraction PDF equation (6). The possibility of deriving the CMC equations from the PDF equation (2) without using (1) needs to be stressed here: in PDF models only the PDF equation (but not the scalar transport equation) is modelled. If a PDF model is compliant with the PDF equation then the consequence of the PDF equation - the unclosed CMC equations can be used in the analysis of the model. One may note that the instantaneous values $Y, N$, etc. are not necessarily defined in PDF models. Thus all correlations in the $\mathrm{CMC}$ equations must be defined in terms of the joint PDF $P_{Y}$ and coefficients of the PDF equations (the conditional expectations $\mathbf{u}_{Y}$ and $\left.N_{Y}^{(i j)}\right)$. For example,

$$
\begin{gathered}
\left\langle\mathbf{u} Y^{(i)} \mid Z\right\rangle=\left\{\mathbf{u}_{Y} Y^{(i)}\right\}_{Z} \\
\left\langle\mathbf{u}^{\prime}\left(Y^{(i)}\right)^{\prime} \mid Z\right\rangle=\left\{\mathbf{u}_{Y} Y^{(i)}\right\}_{Z}-\left\{\mathbf{u}_{Y}\right\}_{Z} Q^{(i)}, \quad Q^{(i)}=\left\{Y^{(i)}\right\}_{Z} \\
\left\langle D \nabla\left(Y^{(i)}\right)^{\prime} \cdot \nabla Z \mid Z\right\rangle=\left\{N_{Y}^{(i 0)}\right\}_{Z}-\frac{\partial Q^{(i)}}{\partial Z} N_{Z}, \quad N_{Z}=\left\{N_{Y}^{(00)}\right\}_{Z} \\
\left\langle N^{\prime}\left(Y^{(i)}\right)^{\prime} \mid Z\right\rangle=\left\{N_{Y}^{(00)} Y^{(i)}\right\}_{Z}-N_{Z} Q^{(i)}, \quad \Theta^{(i j)}=\left\{Y^{(i)} Y^{(j)}\right\}_{Z}-Q^{(i)} Q^{(j)}
\end{gathered}
$$


where the curly brackets subscripted by " $Z$ " denote the following integral

$$
\{(\cdot)\}_{Z} \equiv \frac{1}{P_{Z}} \int(\cdot) P_{Y} d Y^{(1)} \ldots d Y^{(n)}
$$

In this section, we mainly consider simultaneous mixing of only two scalars and use the following notation $Z$ and $Y$. The scalar $Z$, as it is noted above, represents the mixture fraction while the scalar $Y$ is reactive. In order to keep notations simple we denote: $Z=Y^{(0)}, Y=Y^{(1)}, W^{(0)}=0, W=W^{(1)}$ $Q \equiv\langle Y \mid Z\rangle$ and $\Theta \equiv\langle\theta \mid Z\rangle, \theta \equiv\left(Y^{\prime}\right)^{2}, Y^{\prime} \equiv Y-Q$. The equation for the conditional variance $\Theta=\Theta^{(11)}$ takes the form

$$
\begin{gathered}
\frac{\partial \Theta \overline{\boldsymbol{\rho}} P_{Z}}{\partial t}+\boldsymbol{\nabla} \cdot\left(\langle\mathbf{u} \theta \mid Z\rangle \overline{\boldsymbol{\rho}} P_{Z}\right)=\frac{\partial}{\partial Z}\left[N_{Z} \overline{\boldsymbol{\rho}} P_{Z} \frac{\partial \Theta}{\partial Z}-\Theta \frac{\partial N_{Z} \overline{\boldsymbol{\rho}} P_{Z}}{\partial Z}+J_{\theta}\right]+ \\
+2 \overline{\boldsymbol{\rho}} P_{Z}\left[\Psi_{N}+\Psi_{V}+\Psi_{W}-\Psi_{D}\right] \\
\Psi_{N} \equiv\left\langle N^{\prime} Y^{\prime} \mid Z\right\rangle \frac{\partial^{2} Q}{\partial Z^{2}}, \quad \Psi_{D} \equiv\left\langle D\left(\nabla Y^{\prime}\right)^{2} \mid Z\right\rangle \\
\Psi_{V} \equiv-\left\langle\mathbf{u}^{\prime} Y^{\prime} \mid Z\right\rangle \cdot \nabla Q, \quad \Psi_{W} \equiv\left\langle W^{\prime} Y^{\prime} \mid Z\right\rangle
\end{gathered}
$$

The conventional CMC closures are obtained by neglecting the $J$ terms. The justification for this closure can be found in Ref. [5]. Note that compliance with the unclosed CMC equations does not guarantee similarity with CMC closures.

\subsection{Analysis of the long-time asymptote of the conditional generation term}

For the purposes of analysing joint mixing properties of $Z$ and $Y$, the $W$ reaction term is deemed to be switched off for the duration of mixing although $Z$ and $Y$ are not necessarily linked by a linear dependence. Thus, for the duration of mixing, the scalars are governed by the equations

$$
\begin{gathered}
\rho \frac{\partial Z}{\partial t}+\rho \mathbf{u} \cdot \nabla Z-\nabla \cdot(\rho D \nabla Z)=0 \\
\rho \frac{\partial Y}{\partial t}+\rho \mathbf{u} \cdot \nabla Y-\nabla \cdot(\rho D \nabla Y)=0
\end{gathered}
$$

but are constrained by different initial conditions. If at initial moment $t_{0}, Y$ is a deterministic function of $Z$, so that $Y=f(Z)$ and if $f$ is not a linear function, turbulent mixing would result in a certain level of fluctuations $Y^{\prime} \equiv Y-Q$ appearing around conditional mean $Q \equiv\langle Y \mid Z\rangle$. The assumption of initial deterministic dependence between $Y$ and $Z$ is made for more transparent illustration of the problem to be studied: if initially $Y=f(Z)+Y_{0}^{\prime}$ then initial 
conditional fluctuations $Y_{0}^{\prime}$ would eventually disappear while the generated $Y^{\prime}$ would attain the same level. The problem of increasing the dimension of the initial manifold was considered by Pope [6] for multiple species. That consideration is primarily concerned with short-time topological bahaviour while we perform quantitative analysis of the long-time (compared to the Kolmogorov time scale) asymptote for the conditional generation but deal only with two scalars $Y$ and $Z$.

In a locally homogeneous case, when the turbulent transport in physical space is neglected, the equations for conditional variance takes the form

$$
\frac{\partial \Theta}{\partial t}=2 \Psi_{N}-2 \Psi
$$

Assuming that $f$ is relatively graduate function of $Z$, we expand $f$ into Taylor series in the vicinity of a certain point of interest $Z_{0}$. Since the generation of conditional fluctuations is a rather local process with small-scale fluctuations playing a most significant role (note that the small-scale component of turbulent fluctuations is most significant for the dissipation), we need only local representation of $f$. The first and the second terms in the expansion $f=f_{0}+f_{0}^{(\prime)}\left(Z-Z_{0}\right)+\ldots$ do not affect the level of conditional fluctuations and can be locally put to zero without loss of generality $f_{0}=f_{0}^{(\prime)}=0$. Indeed, a new scalar $Y+a_{1} Z+a_{0}$ would have the same conditional variance but $f_{0}$ and $f_{0}^{(\prime)}$ can be set to zero by a proper choice of the constants $a_{1}$ and $a_{0}$. Thus, initially, the scalar $Y$ is represented by the deterministic quadratic dependence $Y=f_{0}^{(\prime \prime)}\left(Z-Z_{0}\right)^{2} / 2$.

First, we note that $Q \equiv\langle Y \mid Z\rangle$ does not remain the same and evolves. The parameters that can be responsible for this process are the average dissipation of energy $E_{0}$, the average scalar dissipation $\bar{N}_{0}$, the initial parameter $f_{0}^{(\prime \prime)}$ and the elapsed time $t-t_{0}$. Generally, the average values should be averaged in the vicinity of the point of interest, for example, $\bar{N}_{0} \equiv\left\langle D(\nabla Z)^{2} \mid Z_{0}\right\rangle$. The dimensionally consistent evolution of $Q$ is given by $Q-Q\left(t_{0}\right)=\operatorname{const} \bar{N}_{0}$ $f_{0}^{(\prime \prime)}\left(t-t_{0}\right)$. The 1 st order CMC equation (4) determines that the consistent value for the constant is 1 and

$$
Q-Q\left(t_{0}\right)=\bar{N}_{0} f_{0}^{(\prime \prime)}\left(t-t_{0}\right)+\ldots
$$

We investigate the evolution of the scalar $Y$ assuming that the macro-parameters $E_{0}, \bar{N}_{0}$ and $f_{0}^{(\prime \prime)}$ do not change much within the observation time period. Considering evolution the second derivative of $Q$ is relatively slow, we can replace $f^{(\prime \prime)}$ by $Q^{(\prime \prime)}=\partial^{2} Q / \partial Z^{2}$ evaluated at $Z=Z_{0}$.

Initially, the conditional variance $\Theta$ grows from its zero value but, when production of conditional fluctuations becomes balanced by their dissipation, $\Theta$ becomes time-independent. It can be seen that the parameters $E_{0}, \bar{N}_{0}$ and $Q^{(\prime \prime)}$ 
are not sufficient to define time-independent $\Theta$ so that another time scale, $\tau_{0}$, must be introduced

$$
\Theta=\tau_{0}^{2}\left(\bar{N}_{0} Q_{0}^{(\prime \prime)}\right)^{2}, \quad Q_{0}^{(\prime \prime)} \equiv \frac{\partial^{2} Q}{\partial Z^{2}}
$$

Effectively, this equation serves as a definition for a new scale $\tau_{0}$ and, thus, does not need a dimensionless constant multiplier. Arguably, since $\Theta$ is significantly smaller than $\left\langle y^{2}\right\rangle$ where $y \equiv Y-\langle Y\rangle$, the time $\tau_{0}$ must be smaller than the time macro-scale and, presumably, $\tau_{0}$ belongs to the inertial interval. In the rest of this section, we will give physical interpretations for the time scales introduced here. The general expression for the conditional dissipation $\Psi_{D}$ can written in the form

$$
\Psi_{D}=\frac{\Theta}{\tau_{D}}
$$

This equation simply reflects that $\Psi_{D}$ must be proportional to $\Theta$ and defines the dissipation time $\tau_{D}$. Since the conditional dissipation term is balanced by the conditional generation term $\Psi_{N}=\Psi_{D}$ for $\Theta$ given by (12), the conditional generation term becomes

$$
\Psi_{N}=\tau_{N}\left(\bar{N}_{0} Q_{0}^{(\prime \prime)}\right)^{2}, \tau_{N} \equiv \frac{\tau_{0}^{2}}{\tau_{D}}
$$

In order to evaluate the characteristic generation time $\tau_{N}$, we substitute $Y=$ $Q(Z, t)+Y^{\prime}$ into equations (8) and (9) while taking into account (11)

$$
\rho \frac{\partial Y^{\prime}}{\partial t}+\rho \mathbf{u} \cdot \nabla Y^{\prime}-\nabla \cdot\left(\rho D \nabla Y^{\prime}\right)=\rho N^{\prime} f_{0}^{(\prime \prime)}
$$

where the scalar dissipation $N \equiv D(\nabla Z)^{2}$ is decomposed into $N=\bar{N}_{0}+$ $N^{\prime}$. Pope [6] considered short-time behaviour of this equation (with multiple scalars involved) and demonstrated that the fluctuations of scalar dissipation are likely to increase the dimensionality of the accessed composition space. This, in our terms, means "generate conditional fluctuations". Here we are interested in quantifying the long-time (compared to the Kolmogorov time scale $\tau_{K}$ ) rate of increase of the conditional variance. If we neglect the diffusion term that is responsible for dissipation of the fluctuations and introduce the Lagrangian derivative $d / d t \equiv \partial / \partial t+\mathbf{u} \cdot \boldsymbol{\nabla}$, equation (15) takes the form $d Y^{\prime} / d t=N^{\prime} f_{0}^{(\prime \prime)}$. The short-time $\Delta t \ll \tau_{K}$ solution for this equation is, obviously, given by

$$
Y^{\prime}=N^{\prime} f_{0}^{(\prime \prime)} \Delta t
$$

while the long-time asymptote is given by the Lagrangian integral

$$
Y^{\prime}=f_{0}^{(\prime \prime)} I^{\prime}, \quad I \equiv \int_{t_{0}}^{t} N d t, \quad I^{\prime}=I-\bar{N}_{0}\left(t-t_{0}\right)=\int_{t_{0}}^{t} N^{\prime} d t
$$


The integral $I^{\prime}$ represents a stochastic value with a zero mean and the following long-time asymptote of its variance

$$
\left\langle\left(I^{\prime}\right)^{2}\right\rangle \rightarrow 2 \tau_{N}^{\circ} \bar{N}_{0}^{2}\left(t-t_{0}\right), \tau_{N}^{\circ} \equiv \int_{0}^{\infty}(K(t)-1) d t, \quad K(\Delta t) \equiv \frac{\left\langle N\left(t_{0}\right) N\left(t_{0}+\Delta t\right)\right\rangle}{\bar{N}_{0}^{2}}
$$

Thus the rate of increase of $\Theta=\left\langle\left(Y^{\prime}\right)^{2}\right\rangle$ is given by $2 \tau_{N}^{\circ}\left(\bar{N}_{0} f_{0}^{(\prime \prime)}\right)^{2}$ so that $\tau_{N}=\tau_{N}^{\circ}$ and $\tau_{N}^{\circ}$ defined in (18) represents a physical interpretation of the time $\tau_{N}$ introduced in (14).

At this point we need to stress the approximate character of equating $\tau_{N}$ and $\tau_{N}^{\circ}$. In our consideration, we separate the generation process from dissipation by neglecting the diffusion term in (15) that is responsible for the conditional dissipation rate estimated by $2 \Theta / \tau_{D}$. In fact, generation and dissipation interfere with each other and $\tau_{N}^{\circ}$ provides an estimation rather than an exact value for $\tau_{N}$. Thus, it is more accurate to conclude that $\tau_{N} \sim \tau_{N}^{\circ}$ pointing to the link between two scales than expect that $\tau_{N}=\tau_{N}^{\circ}$ exactly. Note that fast chemical reactions can effectively terminate the correlation suggested above and this would reduce the value of $\tau_{N}$. Using a single dissipation time, $\tau_{D}$, to characterise the conditional dissipation is also a significant simplification. The fluctuations generated by $N^{\prime}$ have a wide spectrum of scales with excessive presence of smaller scales compared to the conventional turbulent cascade. Thus a particular disturbance with a certain characteristic time scale would have a dissipation scale of the same order and the overall dissipation of conditional fluctuations is characterised by a set of time scales rather than a single scale. The time scale $\tau_{D}$ is effective scale that corresponds to a given spectrum of the generated fluctuations.

We should note that linking $\tau_{N}$ to $\tau_{N}^{\circ}$ is supported by the refined Kolmogorov theory of the inertial interval. These arguments [14] are briefly presented below. In the beginning of this section we considered the case when $Y$ is a smooth function of $Z$. Now we analyse the opposite case when non-zero values of $Y$ can be observed only in the vicinity of $Z=Z_{0}$. Thus, the initial dependence $Y=f(Z)$ is very sharp and is, effectively, the delta-function $Y=\delta\left(Z-Z_{0}\right)$ at $t=t_{0}$. According to the 1 st order CMC, the solution of this problem in given by the Gaussian function

$$
Q=F_{G}\left(\bar{N}_{0} \Delta t, Z-Z_{0}\right), \quad F_{G}(T, Z) \equiv \frac{1}{\sqrt{4 \pi T}} \exp \left(-\frac{Z^{2}}{4 T}\right)
$$

where $\Delta t \equiv t-t_{0}$. As one can see, it is the average value of $N$ determines the solution. If the characteristic scale of a process belongs to inertial interval a partial average of $N$ over the volume that corresponds to this scale must replace the mean value $\bar{N}_{0}$. For Lagrangian-type statistics, it is logical to use the partial average of $N$ over a corresponding time interval instead of the volume average [14]. The partial time average $N_{\Delta t}$, which is given by 
$N_{\Delta t}=I / \Delta t$ where the integral $I$ is defined in (17), replaces $\bar{N}_{0}$ in (19) and $Y=F_{G}\left(I, Z-Z_{0}\right)$. We can represent $I=<I>+I^{\prime}=\bar{N}_{0} \Delta t+I^{\prime}$ and expand the function $F_{G}$ into a series

$$
Y=F_{G}\left(\bar{N}_{0} \Delta t, Z-Z_{0}\right)+\frac{\partial F_{G}\left(I, Z-Z_{0}\right)}{\partial I} I^{\prime}=Q+\frac{\partial^{2} Q}{\partial Z^{2}} I^{\prime}, \quad Y^{\prime}=\frac{\partial^{2} Q}{\partial Z^{2}} I^{\prime}
$$

Here we use $Q$ given by (19) and take into account that $\partial F_{G} / \partial I=\partial^{2} F_{G} / \partial Z^{2}$. As one can see determination of $Y^{\prime}$ in (17) and (20) are very similar and this corresponds to $\tau_{N}=\tau_{N}^{\circ}$.

Accurate determining the time scales from experimental or DNS results is a challenging task. In experiments, non-linear initial dependence between $Y$ and $Z$ is created by chemical reactions. Thus, in experiments, it is quite difficult to separate the influence of mixing from that of chemical reactions. DNS allow to investigate influence of various factors autonomously and, when needed, trace the Lagrangian characteristics but typical Reynolds numbers are not high enough to reliably investigate properties of the inertial interval of turbulence. The properties of the inertial interval are largely responsible for the value $\tau_{N}^{\circ}$. The values $\tau_{N}$ and $\tau_{D}$ have been determined by Direct Numerical Simulations (DNS) of a reacting flow in [15] where a different strategy of closuring the term $\Psi_{N}$ has been pursued. According to [15], $\tau_{D}$ is nearly 4 times smaller than the conventional dissipation time scale (time macro-scale) and $\tau_{N}^{\circ}$ is significantly less than the Kolmogorov time scale. For $\tau_{D}$ being noticeably smaller than the macro-scale is expected since the disturbances generated by the fluctuations of the scalar dissipation have a broad range of characteristic scales and each scale has it own dissipation time. It is clear that, on average, these disturbances would have a surrogate (or effective) dissipation time $\tau_{D}$ less than that of the largest scales in turbulence.

Although it could be the case that, for moderate Reynolds numbers, $\tau_{N}$ is smaller than the Kolmogorov scale as it is illustrated below, it would be rather premature to link $\tau_{N}$ to the Kolmogorov time scale. If $\tau_{N}$ were linked to the Kolmogorov time scale, the conditional fluctuations would not appear at the limit of high Reynolds numbers and this seems to be most unlikely. The conventional independence of the large-scale properties of turbulence on Re in large Reynolds number flows requires that $\tau_{N}(\mathrm{Re}) \rightarrow$ const $>0$ as $\mathrm{Re} \rightarrow \infty$, although the rate of convergence is likely to be very slow since the convergence of fluctuating properties of the scalar dissipation to the presumed universal laws of the inertial interval at the limit of $\operatorname{Re} \rightarrow \infty$ is also very slow. The time $\tau_{N}$ is linked to $\tau_{N}^{\circ}$ and the integral (18) determining $\tau_{N}^{\circ}$ has two major components: integral over the viscous scales and integral over inertial interval. For moderate Reynolds numbers, the integral over viscous scales may be dominant and this is observed in DNS. As the Reynolds number increases, the relative contribution of the viscous scales decreases and we expect that, at high Reynolds numbers, both $\tau_{N}$ and $\tau_{N}^{\circ}$ are determined by the properties 
of the inertial interval. Relatively small values of $\tau_{N}$ in [15] are, probably, due to the influence of chemical reactions.

\section{Generation of conditional fluctuations in PDF models}

In this section we estimate the generation term $\Psi_{N}$ in the common PDF mixing models. The conditions are implied the same as in Section 2.2.

\subsection{IEM}

The mixing term that corresponds to IEM (Interactions by Exchange with the Mean, [16]) model takes the form

$$
\frac{d Y^{(i)}}{d t}=\frac{\left\langle Y^{(i)}\right\rangle-Y^{(i)}}{\tau_{S}}
$$

where $\tau_{S}$ is selected to match the expected physical dissipation rate of the mixture fraction $\langle N\rangle=\left\langle z^{2}\right\rangle / \tau_{S}$ and $z \equiv Z-\langle Z\rangle$. If, initially, $Y=f(Z)$, the IEM mixing would shrink the $Y-Z$ plane in both directions but would not generate any dispersion of $Y$ around $f(Z)$. In homogeneous IEM mixing $Y$ remains a deterministic time-evolving function of $Z$ (i.e. $Y=f(Z, t)$ ). Thus, it is obvious that the conditional generation term in IEM mixing model

$$
\Psi_{N}=0
$$

does not comply with its expected physical value.

\subsection{Modified Curl's model}

In the various versions of the Curls model $[17,18]$, couples of particles are selected randomly and independent from each other and the scalar values assigned to the couple $Y_{(1)}^{(i)}$ and $Y_{(2)}^{(i)}$ reset according to the equation

$$
\dot{Y}_{(1)}^{(i)}=Y_{c}^{(i)}+\beta Y_{d}^{(i)}, \quad \dot{Y}_{(2)}^{(i)}=Y_{c}^{(i)}-\beta Y_{d}^{(i)}
$$

where

$$
Y_{c}^{(i)} \equiv \frac{Y_{(1)}^{(i)}+Y_{2}^{(i)}}{2}, \quad Y_{d}^{(i)} \equiv \frac{Y_{(1)}^{(i)}-Y_{(2)}^{(i)}}{2}
$$

the "acute" symbol indicates new values and $\beta$ is, generally, a random number that is independent of the particle scalar values and $\beta=0$ corresponds to 
complete mixing and $\beta=1$ corresponds to no mixing at all. In Curl's model only a certain fraction of particles are mixed at a given mixing step but we may formally force all particles to form couples (assuming the number of particles is even) and put $\beta=1$ for the couples that do not mix. In old Curl's model $\beta$ can take only values 0 and 1 . In modified Curl's models $\beta$ may have various distributions although $\beta$ is chosen to satisfy $0 \leq \beta \leq 1$ for every couple.

As in the previous analysis we assume that $Y=f(Z)$ where $f$ is a deterministic function (if this is not true we can put $Y=f(Z)+Y^{\prime}$ where the conditional fluctuations dissipate and do not contribute to the production term). By introducing $z_{c} \equiv Z-Z_{c}$ and expanding the function $f$ into the Taylor series at $Z=Z_{c}$ so that $Y=f_{c}+f_{c}^{(\prime)} z_{c}+f_{c}^{(\prime \prime)} z_{c}^{2} / 2+\ldots$, we obtain

$$
\Delta Y_{(p)} \equiv \dot{Y}_{(p)}-f\left(\dot{Z}_{(p)}\right)=\left(1-\beta^{2}\right) Z_{d}^{2} f_{c}^{(\prime \prime)} / 2+\ldots
$$

Here, the subscript " $(p)$ " indicates different particles and can be omitted without causing confusion. Curl's model is not local and by using the Taylor expansion of $f$ we implicitly assume that the function $f$ is weak. The average value of $Z_{d}^{2}$ is linked to and the dissipation rate and the variance change over the time interval $\Delta t$ (that corresponding to the mixing step) by

$$
\langle\gamma\rangle\left\langle Z_{d}^{2}\right\rangle=\left\langle Z^{2}\right\rangle-\left\langle\dot{Z}^{2}\right\rangle=2\langle N\rangle \Delta t, \quad \gamma \equiv\left(1-\beta^{2}\right)
$$

At this point, it is convenient to introduce $z \equiv Z-\langle Z\rangle$ with the same set of indices: $z_{d}=Z_{d}, \quad z_{c}=Z_{c}-\langle Z\rangle, \dot{z}=\dot{Z}-\langle Z\rangle$, etc and note the following identities

$$
\left\langle Z_{d}^{2}\right\rangle=\frac{\left\langle z^{2}\right\rangle}{2},\left\langle z_{c}^{2}\right\rangle=\frac{\left\langle z^{2}\right\rangle}{2},\left\langle z^{2}\right\rangle=\left\langle z_{c}^{2}\right\rangle+\left\langle\beta^{2}\right\rangle\left\langle Z_{d}^{2}\right\rangle,\left\langle Z_{d}^{4}\right\rangle=\frac{\left\langle z^{4}\right\rangle+3\left\langle z^{2}\right\rangle^{2}}{8}
$$

that can be easily obtained due to $\left\langle Z_{d}\right\rangle=\langle z\rangle=\left\langle z_{c}\right\rangle=0$, statistical independence of $\beta, \quad z_{(1)}$ and $z_{(2)}$ and also the equation $\left\langle z_{c} z_{d}\right\rangle=\left\langle z_{(1)}^{2}\right\rangle-\left\langle z_{(2)}^{2}\right\rangle=0$ where the subscripts "(1)" and "(2)" denote different particles. The the obvious consequence of these equations $\langle\gamma\rangle\left\langle z^{2}\right\rangle=4\langle N\rangle \Delta t$ indicates a link between the mean value of $\gamma$ and $\Delta t$

$$
\langle\gamma\rangle=4 \Delta t / \bar{\tau}_{D}, \quad \bar{\tau}_{D} \equiv\left\langle z^{2}\right\rangle /\langle N\rangle
$$

where $\bar{\tau}_{D}$ is the unconditional dissipation time. Note that, to the leading order of the expansion, $\langle\Delta Y\rangle$ - the average change of $Y$ over the time $\Delta t$ is consistent with the 1st order CMC prediction

$$
\langle\Delta Y\rangle=f_{c}^{(\prime \prime)}\langle N\rangle \Delta t
$$

Since Curl's mixing is not local, this consistency is valid only when $f_{c}^{(\prime \prime)}$ is nearly constant in the whole domain under consideration. 
The conditional variance appears to be non-zero after the Curl's mixing step

$$
\left\langle\left(\Delta Y^{\prime}\right)^{2}\right\rangle=\frac{\left(f_{c}^{(\prime \prime \prime}\right)^{2}}{4}\left(\left\langle\gamma^{2}\right\rangle\left\langle Z_{d}^{4}\right\rangle-\langle\gamma\rangle^{2}\left\langle Z_{d}^{2}\right\rangle^{2}\right)=\left(f_{c}^{(\prime \prime \prime}\right)^{2}\langle N\rangle^{2} \Delta t^{2}\left(\varphi_{\gamma} \varphi_{d}-1\right)
$$

where

$$
\varphi_{\gamma} \equiv \frac{\left\langle\gamma^{2}\right\rangle}{\langle\gamma\rangle^{2}}, \quad \varphi_{d} \equiv \frac{\left\langle Z_{d}^{4}\right\rangle}{\left\langle Z_{d}^{2}\right\rangle^{2}}=\frac{\varphi z}{2}+\frac{3}{2}, \quad \varphi_{Z} \equiv \frac{\left\langle z^{4}\right\rangle}{\left\langle z^{2}\right\rangle^{2}}
$$

Considering that the increase in the conditional variance occurs over time $\Delta t$, we note consistency with equation (14) provided the generation time is given by

$$
\Psi_{N}=\tau_{N}\left(\langle N\rangle Q^{(\prime \prime)}\right)^{2}, \quad \tau_{N}=\Delta t \frac{\varphi_{\gamma} \varphi_{d}-1}{2}
$$

The dissipation time for conditional fluctuations coincides with the overall dissipation time: $\tau_{D}=\bar{\tau}_{D}$ Since the generation time is a physical parameter, it must stay finite as $\Delta t \rightarrow 0$. This means that the parameter $\varphi_{\gamma} \sim 1 / \Delta t$ must become larger and larger. (note also that $\langle\gamma\rangle \sim \Delta t / \bar{\tau}_{D}$ ). The equation (30) that examines consistency with the required physical rate for generation of conditional fluctuations is not local and depends on the flatness factor $\varphi_{Z}$ (since $\varphi_{d}$ depends on $\varphi_{Z}$ ). Although this can be expected due to non-local nature of Curl's mixing, the correct selection of the model parameters can be complicated by the well-known feature of many versions of Curl's model to increase $\varphi_{Z}$ with time. It should be noted that the evaluation of $\tau_{N}$ given above is valid only for weak functions $f(Z)$ due to the non-local character of Curl's model. It seems that, practically, Curl's model tend to overpredict generation of conditional fluctuations.

The effective value of the instantaneous scalar dissipation can be determined by comparison with the fast chemistry limit that specifies the chemical rate $W=-N \partial^{2} Y_{e} / \partial Z^{2}$ that is required to counteract mixing and keep the reactive scalar close to its equilibrium value $Y_{e}(Z)$. Considering that, according to (24), $W=-\Delta Y / \Delta t=\gamma Z_{d}^{2} f^{(\prime \prime)} /(2 \Delta t)$ we note that the instantaneous value

$$
N_{\text {eff }}=\gamma \frac{Z_{d}^{2}}{2 \Delta t}=\frac{\gamma}{\langle\gamma\rangle} \frac{Z_{d}^{2}}{\left\langle Z_{d}^{2}\right\rangle}\langle N\rangle
$$

effectively represents the instantaneous value of the scalar dissipation in Curl's mixing. The average value of $N_{\text {eff }}$ is, obviously, $\langle N\rangle$. It should be noted that if the reaction zone becomes thin compared to the macroscale of the mixture fraction, the present analysis is no longer applicable. Hence, Curl's model is not flamelet-consistent at smaller scales. 


\subsection{The EMST model}

The EMST model [19] is based on constructing so called Euclidean Minimal Spanning Tree where each of the particles interacts only with its neighbors in a way that the tree shrinks in the composition space. The process of shrinking by itself does not generate conditional fluctuations and, without intermittency, the model would have a zero conditional generation term. The practical version of the EMST model involves imitation of intermittent behaviour: the particles are divided into two subsets, passive and active. Only the active particles form the evolving tree while the passive particles do not change their scalar values during the mixing step. Particles are allowed to randomly move between the subsets and this process is characterised by a certain residence time that is denoted here by $\tau_{g}$. If, initially, $Y=f(Z)$ the active particles will move from the line $Y=f(Z)$ while passive particles will not and this, obviously, creates fluctuations around conditional mean and $\Theta>0$. Although assessment of the generation term is not easy due to the complexity of EMST algorithm, some estimations can be obtained using the properties of EMST mixing. First, EMST is a local model and the function $f$ can be expanded into a series at a point of interest, say $Z_{0}$. Second, EMST preserves any linear dependence between the scalars $Y=a_{1} Z+a_{0}$ and the function value and its first derivative can be changed by adding $a_{1} Z+a_{0}$ without changing the conditional fluctuations. Hence, as it is in the real turbulence, the second derivative of the function, $f_{0}^{(\prime \prime)}$, is responsible for the generation of the conditional fluctuations. The intensity of EMST mixing should match the dissipation rate $\bar{N}_{0}$ for the scalar $Z$. Using the same dimensional arguments as in Section 2.2, we may write

$$
\Psi_{N}=c_{g} \tau_{g}\left(\bar{N}_{0} f_{0}^{(\prime \prime)}\right)^{2}
$$

where $c_{g}$ is constant and it is taken into account that EMST mixing has its own characteristic time $\tau_{g}$. Note that this estimate of the generation term is obtained only for long-time asymptote so that the details of the EMST mixing do not need to be taken into account directly. The constant $c_{g}$ is not likely to be universal. If initially, or at a certain moment $Y=Q(Z)+Y^{\prime}$ where $Q \equiv\langle Y \mid Z\rangle$ and $Y^{\prime} \neq 0$, the EMST evolutions of fields $Y_{1}=Q$ and $Y_{2}=Y^{\prime}$ are not independent unlike in physical turbulent mixing. Hence, the intensity of conditional fluctuations generated by $Q(Z)$ (and the constant $c_{g}$ ) is dependent on $Y^{\prime}$ in EMST mixing. Practically, the EMST model seems to underpredict generation of conditional variance. EMST is known to be consistent with the fast chemistry limit and the flamelet model. The binary random process that takes either zero value (for the particles from the passive subset) or the other positive value determined by intensity of mixing (for the particles from the active subset ) plays the role of instantaneous scalar dissipation in intermittent EMST mixing. 


\subsection{Basic MMC models}

MMC models $[7,8]$ introduce additional reference variables and require the surrogate mixing to be localised in the reference space. The simplest version of probabilistic MMC has a single mixture fraction-like reference variable $\xi$. The fluctuations of the simulated scalars induced by fluctuations of $\xi$ are called major fluctuations. In probabilistic MMC, the scalars are allowed to fluctuate around their conditional expectations $\bar{Y}^{(i)} \equiv\left\langle Y^{(i)} \mid \xi\right\rangle$ and these fluctuations $Y^{(i)}-\bar{Y}^{(i)}$ called the minor fluctuations simulate the other fluctuations present in a turbulent flow. The minor fluctuations are dissipated by minor dissipation operator $d Y^{(i)} / d t=S\left[Y^{(i)}\right]$ that should not affect the conditional means $\left\langle S\left[Y^{(i)}\right] \mid \xi\right\rangle=0$. This constraint enforces the localness of the surrogate mixing which is used our analysis. Note that this localness can be compromised by the overall non-local nature of the model (for example, in a Partially Stirred Reactor) but MMC is not likely to perform well under these conditions. The minor operator is characterised by the minor dissipation time $\tau_{S}$. The conventional models IEM and Curl's model are obvious candidates to be used in the minor dissipation operator. The case of the operator $S$ represented by IEM $S\left[Y^{(i)}\right]=\left(\bar{Y}^{(i)}-Y^{(i)}\right) / \tau_{S}$ was analysed in [8]. The conditional version of IEM [16] model is called IECM [20]. This is similar to MMC use of the model where the operator $S$ is also conditional. It is shown although the IEM model itself does not generate the conditional variance, the MMC with IEM treatment of the minor fluctuations does increase $\Theta$. The minor dissipation time should be selected to match the expected physical intensity of conditional fluctuations. The analysis is quite difficult mainly due the fact that the operator $S$ controls fluctuations around $\bar{Y}^{(i)}$ while the conditional fluctuations around $Q=\left\langle Y^{(i)} \mid Z\right\rangle$ where $Z$ is the simulated mixture fraction are of physical interest. The latter fluctuations depend on former fluctuations but these fluctuations should not be confused with each other. The analysis [8] determined that the dissipation time for conditional fluctuations is $\tau_{D}=\tau_{S}$ and that the generation term is given by

$$
\Psi_{N}=\tau_{N}\left(\bar{N}_{0} Q_{0}^{(\prime \prime)}\right)^{2}, \quad \tau_{N}=\tau_{S} / 6
$$

For fast chemistry case, the instantaneous value of the scalar dissipation in MMC-IEM mixing is simulated by

$$
N_{\text {eff }}=\langle N\rangle \frac{1+\omega^{2}}{2}
$$

where $\omega$ is the standard Gaussian stochastic value (i.e. $\langle\omega\rangle=0$ and $\left\langle\omega^{2}\right\rangle=1$ ). The model preserves flamelet consistency for the scales that correspond to the major fluctuations but is not consistent with the flamelet model for scales that correspond to the minor fluctuations. 
The case of the minor operator represented by Curl's mixing is much easier to analyse since Curl's mixing explicitly generates the conditional fluctuations. The MMC-Curl mixing is different from conventional Curl's model by allowing mixing only between particles that are close to each other in the $\xi$-space. The equations obtained in Section 3.2 can still be used but $z \equiv Z-\langle Z\rangle$ needs to be replaced by fluctuations $Z^{\prime \prime} \equiv Z-\bar{Z}, \bar{Z} \equiv\langle Z \mid \xi\rangle$ localised in $\xi$-space and $\bar{\tau}_{D}$ needs to be replaced by $\tau_{S}$. Under the standard MMC conditions, the generation rate of the minor fluctuations is the same as the dissipation rate of the major fluctuations that is given by

$$
2 \bar{N}=2 B\left(\frac{\partial \bar{Z}}{\partial \xi}\right)^{2}
$$

where $B$ is the diffusion coefficient in $\xi$-space, while the dissipation rate of the minor fluctuations by Curl's mixing is $2 \Theta_{\xi} / \tau_{S}$, where $\Theta_{\xi} \equiv\left\langle\left(Z^{\prime \prime}\right)^{2} \mid \xi\right\rangle$. Hence,

the quasi-steady level of minor fluctuations is give by $\Theta_{\xi}=\bar{N} \tau_{S}$. Although the level of minor fluctuations $Z^{\prime \prime}$ is, under typical MMC conditions, smaller than the level of major fluctuations determined by $z$, the dissipation rate of minor fluctuations is the same as that for major fluctuations due to having dissipation time $\tau_{S}$ smaller than $\bar{\tau}_{D}$. The equations (30) linking the conditional generation rate to the dissipation rate and other parameters remain the same (although we can estimate $\varphi_{d} \approx \varphi_{z} \approx 3$ due to intensive Gaussian generation of the minor fluctuations)

$$
\Psi_{N}=\tau_{N}\left(\bar{N}_{0} Q_{0}^{(\prime \prime)}\right)^{2}, \quad \tau_{N}=\Delta t \frac{3 \varphi_{\gamma}-1}{2}, \varphi_{\gamma} \equiv \frac{\left\langle\gamma^{2}\right\rangle}{\langle\gamma\rangle^{2}}
$$

The subscript " 0 " indicates that analysis is performed in the vicinity of $Z_{0}$ and the model is local. The are, however, some differences that worth noting. The dissipation time for the conditional fluctuations is now determined by the minor dissipation time $\tau_{D}=\tau_{S}$. The values of $\gamma$ need to be increased (compared to the conventional Curl's model) in order to provide a more intense dissipation $\langle\gamma\rangle=4 \Delta t / \tau_{S}$. The mixing couples are localised in $\xi$-space and the MMC-Curl model does not have the problem of unrestricted increase of the flatness factor $\left\langle z^{4}\right\rangle /\left\langle z^{2}\right\rangle^{2}$ that exacerbates the performance of many Curl-type mixing models.

\section{Generalised understanding of the reference conditioning}

Here we consider the MMC model that is represented by the following stochastic Ito equations

$$
\begin{gathered}
d \mathbf{x}=\mathbf{u}(\boldsymbol{\xi}, \mathbf{x}, t) d t \\
d \xi_{k}=A_{k}^{\circ}(\boldsymbol{\xi}, \mathbf{x}, t) d t+b_{k l}(\boldsymbol{\xi}, \mathbf{x}, t) d w_{l}
\end{gathered}
$$




$$
\begin{gathered}
d Y^{(i)}=\left(W^{(i)}(\mathbf{Y})+S\left[Y^{(i)}\right]\right) d t, \\
\left\langle S\left[Y^{(i)}\right] \mid \boldsymbol{\eta}, \mathbf{x}\right\rangle=0, \eta_{i}=\eta_{i}(\boldsymbol{\xi} ; \mathbf{x}, t) \\
A_{k}^{\circ} \equiv A_{k}+\frac{2}{P_{\xi}} \frac{\partial B_{k l}\langle\rho\rangle P_{\xi}}{\partial \xi_{l}}, \quad 2 B_{k l}=b_{k j} b_{l j} .
\end{gathered}
$$

where $S$ is the mixing operator, $W^{(i)}$ specify chemical source terms and $P_{\xi}$ is the PDF of the reference variables $\xi_{j}$. This form of MMC equations is somewhat more general than its original formulation [7] since the conditioning variables $\boldsymbol{\eta}=\left\{\eta_{1}, \ldots, \eta_{n_{c}}\right\}$ are not necessarily the same as the reference variables $\boldsymbol{\xi}=\left\{\xi_{1}, \ldots, \xi_{n_{r}}\right\}$. We assume that $n_{c} \leq n_{r}$ since the excessive $\left(n_{c}>n_{r}\right)$ conditioning variables would lay within $n_{r}$-dimensional manifold and would not impose additional conditions. The conditioning variables are deemed to emulate certain Lagrangian characteristics of a turbulent flow (that can be dissipation, velocities, etc) while the reference variables are used to assist simulations. The physical properties emulated by the conditioning variables can be called observed of traced properties to distinguish them from the other characteristics of turbulence that are not directly simulated in the model. It is implied that the observed characteristics are the ones that strongly affect combustion. Note that additional reference variable do not impose a large computational cost on the model while increasing the dimension of the conditioning space requires a significant increase in the number of particles for proper evaluation of the conditional expectation and this is computationally expensive. The generalised MMC can be transformed into the form that is more close to the original version by introducing the new reference variables $\hat{\boldsymbol{\xi}}=\left\{\eta_{1}, \ldots, \eta_{n_{c}}, \xi_{1}, \ldots, \xi_{n_{r}-n_{c}}\right\}$. The dimension of the new reference space is the same as the dimension of the old reference space given by $n_{c}$. The MMC equations preserve their form but the old coefficients $A_{k}, A_{k}^{\circ}, B_{k l}$ and $b_{k j}$ should be replaced by the new coefficients $\hat{A}_{k}, \hat{A}_{k}^{\circ}, \hat{B}_{k l}$ and $\hat{b}_{k l}$ whose values are specified in ref. [8]. In original MMC [7], all of the variables $\hat{\boldsymbol{\xi}}$ are used for conditioning and $\left\langle S\left[Y^{(i)}\right] \mid \hat{\boldsymbol{\xi}}, \mathbf{x}\right\rangle=0$. If $n_{c}=n_{r}$, the generalised MMC is equivalent to its original formulation, but if $n_{c}<n_{r}$ then $\left\langle S\left[Y^{(i)}\right] \mid \boldsymbol{\eta}, \mathbf{x}\right\rangle=0$ but, in general, $\left\langle S\left[Y^{(i)}\right] \mid \hat{\boldsymbol{\xi}}, \mathbf{x}\right\rangle \neq 0$. Thus, the conditional expectations $\left\langle Y^{(i)} \mid \hat{\boldsymbol{\xi}}, \mathbf{x}\right\rangle$ are, obviously, not the same in both versions. While $\left\langle Y^{(i)} \mid \boldsymbol{\eta}, \mathbf{x}\right\rangle$ are not directly affected by the change in formulation of the operator $S$ but this change affects the fluctuations of $Y^{(i)}$ with respect to their conditional means $\left\langle Y^{(i)} \mid \boldsymbol{\eta}, \mathbf{x}\right\rangle$. These fluctuations alter the conditional means $\left\langle Y^{(i)} \mid \boldsymbol{\eta}, \mathbf{x}\right\rangle$ through interactions with the model coefficients and the non-linear reaction terms $W^{(i)}(\mathbf{Y})$. Thus the generalised model is not identical to the original version, although their treatments of $\left\langle Y^{(i)} \mid \boldsymbol{\eta}, \mathbf{x}\right\rangle$ can be expected to be similar if conditional fluctuations can be neglected.

The generalised formulation of the MMC model is a natural extension of the 
original version. Indeed, practical simulations deal with a number of particles that may be large but finite. In original MMC, mixing between particles is allowed only if the particles are close to each other in $\xi$-x-space and this requires definition of the distance $d$ in the $\xi$ - $x$-space. Generally, the distance between two particles subscripted by "(1)" and "(2)", can be defined by $d^{2}=g^{k l} \Delta \zeta_{k} \Delta \zeta_{l}$ where $\Delta(\cdot)=(\cdot)_{(1)}-(\cdot)_{(2)}, g^{k l}$ represents a metric tensor and $\boldsymbol{\zeta}=\{\boldsymbol{\xi}, \mathbf{x}\}$ represent a vector in the $\xi$-x-space. The definition of the distance affects the performance of the model. In order to demonstrate this, let us for the sake of simplicity consider a homogeneous case and assume that $d^{2}=g^{11} \Delta \xi_{1} \Delta \xi_{1}+g^{22} \Delta \xi_{2} \Delta \xi_{2}$ where reference variables have zero mean and unit dispersion. If $g^{22} \ll g^{11}$ the neighborhood of a point is stretched in the direction of $\xi_{2}$ so that mixing particles must be close in $\xi_{1}$-direction but can be at a substantial distance in $\xi_{2}$-direction. Thus, even if both variables $\xi_{1}$ and $\xi_{2}$ are used as the conditioning variables, a non-isotropic choice of the metric tensor allows to make mixing non-local in the selected directions. A very small value of $g^{22}$ would, effectively, completely exclude $\xi_{2}$ from the list of conditioning variables. The generalised MMC corresponds to the original MMC with the pseudometric in $\xi$-space defined by $d^{2}=\eta_{j}(\boldsymbol{\xi}) \eta_{j}(\boldsymbol{\xi})$.

At this point we need to introduce certain principles that ensure that the generalised MMC achieves its goal of good modelling. Turbulent dispersion without dissipation is considered first. In a physical turbulent flow, we select some of fluid particles and mark them with $Z=1$ while $Z=0$ remain for the rest of fluid particles. Each particle is assigned a certain mass $m=M / n_{p}$ which is small when the total number of fluid particles $n_{p}$ is large (here $M$ represents the total mass in the domain under consideration). One can imagine that these particles are similar to molecules but, unlike molecules, the particles are not engaged in Brownian motion and move with the local velocity of the flow. As in previous sections, $Z \equiv Y^{(0)}$ is a passive scalar not affected by the reactions $W^{(0)}=0$. The values of $Z$ remain constant for each fluid particle. In simulations, this case corresponds to the switched off mixing operator $S[Z]=0$. We require that whatever the initial distribution of the marked particles may be, stochastic variables $\eta_{j}$ should emulate well the nominated Lagrangian physical properties of the turbulent flow. We presume that $\boldsymbol{\eta}(\boldsymbol{\xi}, \mathbf{x} ; t)$, where $\hat{\boldsymbol{\xi}}$ is Markov vector process of dimension of $n_{r}$, can imitate certain Lagrangian physical characteristics of smaller dimension $n_{c}$ (that are, generally, non-Markovian). Proper simulation of Lagrangian statistical properties ensures that the Lagrangian PDF $P_{L}(\boldsymbol{\eta}, \mathbf{x} ; t)$ is adequately represented by the model. In case of a single (say the first) particle in a turbulent flow, its Lagrangian PDF is given by $P_{L}\left(\boldsymbol{\eta}^{\circ}, \mathbf{x}^{\circ} ; t\right)=\left\langle\psi_{(1)}\right\rangle$ where $\psi_{(p)} \equiv \delta\left(\boldsymbol{\eta}(t)_{(p)}-\boldsymbol{\eta}^{\circ}\right) \delta\left(\mathbf{x}(t)_{(p)}-\mathbf{x}^{\circ}\right)$ is the so-called fine-grained PDF and the subscript " $p$ " runs over different particles [25]. If $n_{s}$ equivalent particles are selected, then $P_{L}\left(\boldsymbol{\eta}^{\circ}, \mathbf{x}^{\circ} ; t\right)=\left\langle\Sigma_{p} Z_{(p)} \psi_{(p)}\right\rangle / n_{s}$ where the summation is formally performed over all particles but only the selected particles contribute to the 
sum and $n_{s}=\Sigma_{p} Z_{(p)}$ is the number of selected particles. The integral of the function

$$
\int_{V}\left\langle\sum_{p} m \psi_{(p)}\right\rangle d \boldsymbol{\eta}^{\circ} d \mathbf{x}^{\circ}=M_{V}=\int_{V} P(\boldsymbol{\eta} ; \mathbf{x}, t) \bar{\rho}(\mathbf{x}, t) d \boldsymbol{\eta} d \mathbf{x}
$$

represents the average total mass of all particles within any arbitrary volume $V$ in the $\boldsymbol{\eta}$-x phase space (i.e. $M_{V}$ is the mass of the fluid in this volume). The same mass can be obtained by intergrating the Eulerian Favre (density weighted) PDF times average density $\bar{\rho} \equiv\langle\rho\rangle$ over the same volume, as it is shown on the right-hand side of this equation. Since the volume is arbitrary, we can write $\left\langle\Sigma_{p} m \psi_{(p)}\right\rangle=P\left(\boldsymbol{\eta}^{\circ} ; \mathbf{x}^{\circ}, t\right) \rho\left(\mathbf{x}^{\circ}, t\right)$ and

$$
P_{L}\left(\boldsymbol{\eta}^{\circ}, \mathbf{x}^{\circ} ; t\right)=\frac{1}{n_{s}}\left\langle\sum_{p} Z_{(p)} \psi_{(p)}\right\rangle=\frac{1}{M_{s}}\left\langle Z \mid \boldsymbol{\eta}^{\circ}, \mathbf{x}^{\circ}, t\right\rangle P\left(\boldsymbol{\eta}^{\circ} ; \mathbf{x}^{\circ}, t\right) \rho\left(\mathbf{x}^{\circ}, t\right)
$$

where $M_{s} \equiv m n_{s}=\Sigma_{p} m Z_{(p)}$ is the total mass of the selected particles. Since proper simulation of Lagrangian stochastic properties of the physical parameters $\boldsymbol{\eta}(t)$ ensures adequate simulation of the Lagrangian PDF $P_{L}(\boldsymbol{\eta}, \mathbf{x} ; t)$, the model will simulate well the 1 st order conditional expectations $\bar{Z}(\boldsymbol{\eta}, \mathbf{x}, t)=$ $\langle Z \mid \boldsymbol{\eta}\rangle$ (with arbitrary initial distribution of $Z$ ) and the Eulerian $\operatorname{PDF} P(\boldsymbol{\eta} ; \mathbf{x}, t)$.

The physical dissipation that is inherently present in the turbulent flow would result in mixing of the values $Z$ so that $0 \leq Z \leq 1$ rather than $Z=0$ and $Z=1$. This mixing process is presumed to be local so that the conditional means $\bar{Z}$ remain are not significantly affected by mixing. In MMC model the physical mixing is substituted by a surrogate mixing. Although condition (39) ensures that the mixing does not affect the conditional expectation $\bar{Z}$, the surrogate mixing and physical mixing are, obviously, not equivalent. Mixing of physical fluid particles is allowed only if the particles are very close to each other in physical space so that not only $\boldsymbol{\eta}$ but all other continuous physical parameters are the same (or nearly the same) for both particles. The surrogate mixing allows two particles to be mixed provided the traced values $\boldsymbol{\eta}$ are the same (or sufficiently close). The simulation is aimed at reducing the number of the traced particles compared to the prototype physical flow while keeping this number large enough to adequately simulate the required characteristics. Although it is unlikely that the surrogate mixing can be the same as the physical mixing, the intensity of the surrogate mixing should be selected to match the most important physical parameter - the conditional variance $\Theta_{\eta} \equiv$ $\left\langle\theta_{\eta} \mid \boldsymbol{\eta}^{\circ}\right\rangle$ where $\theta_{\eta} \equiv(Z-\bar{Z})^{2}$. The surrogate mixing can be represented by any scheme that a) preserves the sum of the values $Z_{(p)}$, b) reduces $\max \left(Z_{(p)}\right)$ and increases $\min \left(Z_{(p)}\right)$ c) treats all scalars $Y^{(i)}$ in the same way and d) is local in the $\boldsymbol{\eta}$-X phase space. The examples of good and relatively simple mixing schemes are given by Curl's mixing and IECM. The conditioning variables should be selected in a way that ensures that $\Theta_{\eta}$ is small compared to the 
unconditional fluctuations $\left\langle z^{2}\right\rangle$ (where $z=Z-\langle Z\rangle$ )) since direct mixing of widely dispersed values is not local. In MMC, we label the more detailed simulation of the fluctuations that are related to the conditional values $\bar{Z}$ as major and the less detailed, surrogate simulation of the fluctuations linked to the conditional variance $\Theta_{\eta}$ as minor. The basic principles of generalised MMC modelling are now summarised

(1) The conditional variables $\boldsymbol{\eta}$ with assistance of Markov reference variables emulate as closely as possible certain selected Lagrangian values in a turbulent flow and this ensures the adequate simulation of conditional expectations $\bar{Z}$.

(2) The intensity of surrogate mixing, which is performed by Curl's mixing, IECM or similar models locally in the $\boldsymbol{\eta}$-space, should match the expected physical level of the conditional fluctuations $\Theta_{\eta}$.

(3) The conditioning variables $\boldsymbol{\eta}$ should be selected in a way so that a minimal number of conditioning variables still keeps $\Theta_{\eta}$ relatively small (i.e. $\eta_{1}, \ldots, \eta_{n_{c}}$ represent turbulence parameters that strongly affect the combustion process)

These principles seem to be quite simple and transparent but we should note few complications. First, we have only limited information about 1st and 2nd moment characteristics conditioned on physical values other than the mixture fraction. The conditioning on mixture fraction has been studied extensively but we can not state the same about other possible conditioning variables. Second, in the previous discussion, we presumed that the conditioning variables $\boldsymbol{\eta}$ directly emulate certain characteristics of a turbulent flow. This may be true for velocities, but not for the mixture fraction. Direct emulation of the mixture fraction by, say, $\eta_{0}$ would violate independence and boundedness of the scalars. The emulated value $\eta_{0}$ is not a mixture fraction but a mixturefraction like variable that the simulated mixture fraction and other scalars are mapped into. It is still preferable that the characteristics of $\eta_{0}$ are as close to the characteristics of the mixture fraction $Z$ as possible but the fluctuations $Y^{(i)}-\left\langle Y^{(i)} \mid \eta_{0}\right\rangle$ are not the same as the fluctuations $Y^{(i)}-\left\langle Y^{(i)} \mid Z\right\rangle$ although they are linked to each other. The model parameters must be selected in order to match the physical level of conditional fluctuations $Y^{(i)}-\left\langle Y^{(i)} \mid Z\right\rangle$ as it is discussed in Sections 2, 3 and in Ref [8]. If among conditioning variables, $\eta_{0}$ is a mixture-fraction-like variable while the other variables $\eta_{1}, \eta_{2}, \ldots$ emulate certain physical quantities, the intensity of mixing by the operator $S$ should be chosen to match the physical level of conditional fluctuations with respect to $\left\langle Y^{(i)} \mid Z, \eta_{1}, \eta_{2}, \ldots\right\rangle$ where $Z$ is substituted for $\eta_{0}$ in the list of the conditioning variables. The requirement that not only Lagrangian transitional PDFs but also all stochastic properties of the Lagrangian processes $\boldsymbol{\eta}(t)$ should be emulated may seem excessive. However, under Markov assumptions the transitional probabilities fully determine the process and matching the probabilities is equivalent to simulating the whole stochastic process. In generalised 
MMC, the implicit reference variables (that are not used for conditioning) introduce some memory into the model. Presence of chemical reactions that, generally, are no less than nonlinear functionals of the observed parameters of turbulence, matching the PDFs without matching the process seems unlikely. Hence, complete emulation of the physical processes $\boldsymbol{\eta}(t)$ is targeted in MMC modelling but, at the same time, it is clear that the target has to be compromised to a certain extent in practical modelling. In the following sections we consider examples of the generalised MMC models that illustrate the principles established in this section.

\section{Examples of the generalised MMC models}

\subsection{MMC involving emulation of the dissipation rate}

Assuming that the mixture fraction-type reference and conditioning variable is given by $\xi_{0}=\eta_{0}$, we represent the corresponding dissipation term by

$$
N_{Z} \approx B_{00}\left(\frac{\partial\left\langle Z \mid \eta_{0}\right\rangle}{\partial \eta_{0}}\right)^{2}, \quad B_{00}=\bar{B} \Phi\left(\xi_{1}, \ldots, \xi_{n_{d}}\right), \quad\langle\Phi\rangle=1
$$

where $\bar{B}$ is the value of parameter $B_{00}$ without modelling the fluctuations of the dissipation rate and $N_{Z}$ is the conditional dissipation of the mixture fraction. The reference variables $\xi_{j}=\left\{\xi_{1}, \ldots, \xi_{n_{d}}\right\}$, where $n_{d}=n_{r}-1$ is the number of the dissipation-like reference variables, are presumed to be standard Gaussian (with zero mean and unit dispersion) and, since $\Phi$ is expected to be log-normal, we can write

$$
\begin{gathered}
\Phi=\exp (\phi), \quad \phi=c_{j} \xi_{j}(t)+c_{0} \\
\langle\Phi\rangle=\exp \left(c_{0}+\frac{c_{j} c_{j}}{2}\right)=1, c_{0}=-\frac{c_{j} c_{j}}{2} \\
\left\langle\Phi^{2}\right\rangle=\exp \left(2 c_{0}+2 c_{j} c_{j}\right)=\exp \left(c_{j} c_{j}\right) \\
B_{j \alpha}=\frac{\delta_{j \alpha}}{\tau_{\alpha}}, B_{0 j}=0, A_{\alpha}=\frac{\xi_{\alpha}}{\tau_{\alpha}}+a_{\alpha}
\end{gathered}
$$

where $a_{\alpha}=0$ since the dissipation-related reference variables $\xi_{j}$ are independent of velocity $\mathbf{u}$. In this section, the indices run over $1,2, \ldots, n_{d}$ and sum is not taken over Greek indices. The dissipation correlation

$$
K(t)=\frac{\langle N(0) N(t)\rangle}{\langle N\rangle^{2}} \approx\langle\Phi(0) \Phi(t)\rangle=\langle\exp (\phi(0)+\phi(t))\rangle=\exp (\kappa(t))
$$


where

$$
\kappa(t) \equiv\left\langle\phi^{\prime}(0) \phi^{\prime}(t)\right\rangle=c_{j}^{2} \exp \left(-\frac{t}{\tau_{j}}\right), \phi^{\prime}=\phi-\langle\phi\rangle=\phi-c_{0}
$$

and the sum is taken over index $j=1, \ldots, n_{d}$ in this equation. The fact that the average of the first two moments of $\phi(0)+\phi(t)$ is given by $2 c_{0}$ and $c_{j} c_{j}+$ $c_{0}^{2}+\langle\phi(0) \phi(t)\rangle$ has been used in the derivation. Since $\phi(t)$ represent a linear combination of independent Orstein-Ulhenbeck stochastic processes $\xi_{j}(t)$ with their correlation functions $\exp \left(-t / \tau_{j}\right)$, the correlation $\kappa(t)$ is easily evaluated.

Within the inertial interval $K \sim\left(t / \tau_{M}\right)^{-\varkappa}$ where, as it can be inferred from [21], $\varkappa \approx 0.5$ and $\tau_{M}$ represents the time macroscale. With the use of $s \equiv \ln (t)$ and $s_{(\cdot)} \equiv \ln \left(\tau_{(\cdot)}\right)$ for any subscript $(\cdot)$, we note that

$$
\varkappa\left(s_{M}-s\right) \sim \kappa=c_{j}^{2} \exp \left(-\exp \left(s-s_{j}\right)\right)
$$

If $s_{j}$ are spaced so that $s_{K} \sim s_{n_{d}}<s_{n_{d}-1}<\ldots<s_{2}<s_{1} \sim s_{M}$, where the subscripts " $M$ " and " $K$ " correspond to the time macro- and Kolmogorov scales, the coefficients $c_{\alpha}^{2}$ are given by

$$
c_{\alpha}^{2}=\varkappa\left(s_{\alpha-1}-s_{\alpha}\right)
$$

The cascade model within the inertial interval corresponds to the equidistant steps $\Delta s=s_{\alpha-1}-s_{\alpha}$. Indeed, the cascade of scales is conventionally selected so that the ratio $\tau_{\alpha-1} / \tau_{\alpha}=\exp (\Delta s)$ remains the same and, at $\alpha^{\text {th }}$ level of cascade, the stochastic variable $\xi_{\alpha}$ can intensify or abate the level of dissipation by multiplying the dissipation by a positive random number $\exp \left(c_{\alpha} \xi_{\alpha}\right)$. Practically $\Delta s \sim 1$ produces smooth correlation curves and there is no advantage in having smaller steps. The inertial interval exponent becomes noticeable when $s_{1}-s_{n_{d}}$ reaches 3 and we have 4 (or more) dissipation-like reference variables. This roughly corresponds to the Reynolds number of $\sim 10^{3}$.

The choice of the conditioning variables is now discussed. Klimenko [22] considered MMC model that is similar to the model introduced in this subsection and has all reference variables used for conditioning. That work gave a rather formal proof that matching Lagrangian characteristics of the scalar dissipation ensures compliance with the required rate of generation of conditional fluctuations discussed in Section 2.2. The proof is technically complicated and, if we accept the physical arguments of the present work, is not needed here. The analysis [22] gives some indications that a proper emulation of the dissipation rate should make the simulations of the conditional generation rate to be reaction-independent. Selecting all reference variables as the conditioning ones $\eta_{j}=\xi_{j}$ seems to be excessive and computationally expensive. Although there is a valid argument that, if the set of variables $\xi_{1}, \xi_{2}, \ldots$ represents the turbulent cascade, their values must be the same (or nearly the same) for neighboring points in physical space, we can also treat $\xi_{i}$ as mere mathematical variables 
needed to generate proper stochastic process close to the Lagrangian characteristics of the dissipation. The first obvious choice for a dissipation-like conditioning variable is $\eta_{1}=\Phi$ or, equivalently, $\eta_{1}=\phi$. However, the analysis of dissipation conditioning performed in [12] with the use of DNS seems to demonstrate a significant dispersion around concentrations conditioned on the scalar dissipation rate. Indeed, the scalar dissipation changes rapidly, it is not a good variable to define a locality for the purposes of mixing. It seems that a more conservative dissipation-related variable $\eta_{2}=\tau_{j}^{\varsigma} c_{j} \xi_{j}(t)$ with some $\varsigma>0$ may be used to obtain a more conservative definition of locality.

\subsection{MMC and LES}

The generalised MMC model with explicit modelling of dissipation fluctuations considered in the previous subsection seems to be a natural associate of LES (Large Eddy Simulations). Methodologically, LES resolve most of the velocity and scalar fluctuations but, if the Reynolds number is high, very little of the dissipation fluctuation. The MMC model can effectively simulate the adequate level of the dissipation fluctuations. First we introduce the MMC cell that is expected to be larger than the LES grid size but still small compared to the macroscales of turbulence. A large number of fluid particles is traced in LES calculations and the MMC properties $Z^{(i)}$ and $\xi_{j}$ are assigned to each of the particles. Each MMC cell contains many fluid particles. The cell averages are called cell-resolved values and denoted by the "tilde" while the subcell fluctuations are denoted by the double-prime superscript. The reference mixturefraction like variable is represented by $\eta_{0}=\tilde{\eta}_{0}+\eta_{0}^{\prime \prime}$ where $\tilde{\eta}_{0}=\tilde{Z}_{\text {LES }}$ is the cell averaged mixture fraction $Z_{\mathrm{LES}}$ evaluated from LES and $\eta_{0}^{\prime \prime}$ is modelled by the MMC Markov diffusion process as considered in the previous subsection. The coefficients $B_{00}$ and $A_{0}$ are selected to match the level of subcell fluctuations of the mixture fraction that can be dynamically determined from LES. The coefficient $B_{00}$ is represented by $B_{00}=\tilde{B} \Phi\left(\xi_{1}, \ldots, \xi_{n_{d}}\right)$ where $\tilde{B}$ is the dynamically evaluated cell-resolved dissipation and $\Phi\left(\xi_{1}, \ldots, \xi_{n_{d}}\right)$ is analogous to $\Phi$ from previous subsection but, here, $\Phi$ models only the subcell fluctuations of the dissipation and the process times $\tau_{j}$ range from Kolmogorov to cell-related characteristic times. Since dissipation is not resolved in LES, emulation of dissipation fluctuations is based on the theory of the inertial interval. Note that the mixture fraction $Z_{\mathrm{LES}}$ evaluated by LES is not treated as the "real" mixture fraction - this would violate the independence of scalars. The mixture fraction is assigned to each fluid particle (explicitly or implicitly as a linear combination of $Y^{(i)}$ ) and modelled in accordance with equation (38). Within the cell scales, the conventional definition of locality is compromised to account for subcell fluctuations of the dissipation. The new definition of locality involves both the conditioning variables $\boldsymbol{\eta}$ and the physical coordinates $\mathrm{x}$. 


\subsection{Velocity-like reference variables in $M M C$}

The stochastic formulation of the conventional IEM and IECM models [16,23,20,24] is given by the following equations.

$$
\begin{gathered}
d x_{j}=u_{j} d t, u_{j}=\xi_{j} \\
d \xi_{j}=A_{j}^{\circ} d t+\left(C_{0} \bar{\varepsilon}\right)^{1 / 2} d w_{j}, \quad A_{j}^{\circ}=-a^{\circ} \xi_{j} \\
d Y^{(i)}=W^{(i)}+S\left[Y^{(i)}\right] d t \\
\mathrm{IECM:} S\left[Y^{(i)}\right]=\frac{\left\langle Y^{(i)} \mid \mathbf{u}, \mathbf{x}\right\rangle-Y^{(i)}}{\tau_{S}} ; \mathrm{IEM}: \quad S\left[Y^{(i)}\right]=\frac{\left\langle Y^{(i)} \mid \mathbf{x}\right\rangle-Y^{(i)}}{\tau_{S}}
\end{gathered}
$$

where $\bar{\varepsilon}$ denotes the average dissipation of energy, $C_{0}$ is the Kolmogorov constant and $j$ runs over $1,2,3$. On the face of the problem, it seems that IECM belongs to the class of MMC models with the conditioning and reference variables given by $\eta_{j}=\xi_{j}=u_{j}$ while IEM is a generalised MMC with the reference variables $\xi_{j}=u_{j}$ and without any conditioning variables. A more detailed consideration, however, indicates that the IECM model does not operate in the regime expected for an MMC model. Indeed, since IECM can be treated formally as an MMC model, the MMC techniques [7] indicate that IECM corresponds to the following equation for the conditional mean $\bar{Z}_{u} \equiv\langle Z \mid \mathbf{u}, \mathbf{x}\rangle$

$$
\frac{\partial \bar{Z}_{u}}{\partial t}+\mathbf{u} \cdot \nabla \bar{Z}_{u}+A_{j} \frac{\partial \bar{Z}_{u}}{\partial u_{j}}-\frac{C_{0} \bar{\varepsilon}}{2} \frac{\partial^{2} \bar{Z}_{u}}{\partial u_{j} \partial u_{j}}=\langle S[Z] \mid \mathbf{u}, \mathbf{x}\rangle=0
$$

where $Z=Y^{(0)}$ represents the mixture fraction and $A_{j}$ is linked to $A_{j}^{\circ}$ by (40). In conditional MMC, where $\bar{Z}$ is interpreted as a model for the scalar, the scalar dissipation is given by

$$
\bar{N}=\frac{C_{0} \bar{\varepsilon}}{2} \frac{\partial \bar{Z}}{\partial u_{j}} \frac{\partial \bar{Z}}{\partial u_{j}}
$$

A similar rate for the dissipation of velocity components and the scalar dissipation would correspond to $C_{0} \approx 2 / 3$ while the commonly acceptable value for this constant is around 5 time higher [25]. Thus, if dissipation is determined by equation (56), it would be significantly overestimated by the model. In conventional MMC, the overall dissipation rate is determined by equation (56) while the operator $S$ has a characteristic time of $\tau_{S}$ that is linked to the level of conditional fluctuation. In case of IECM model, the characteristic dissipation time $\tau_{S}$ has to be selected similar to the average dissipation time $\bar{\tau}_{D}$ in order to match the overall dissipation rate. This results in a large level of fluctuations around the averages conditioned on $\mathbf{u}$ and in essentially non-local regime for the mixing operator $S$. 
In this subsection, we assume that spatial scalar transport properties are adequately simulated by model (51)-(53) with the operator $S\left[Y^{(i)}\right]$ represented by IECM (generally, $S$ can be represented by any another mixing model localised in the $\mathbf{u}$-space and complying with $\left.\left\langle S\left[Y^{(i)}\right] \mid \mathbf{u}, \mathbf{x}\right\rangle=0\right)$. We extend the model in order to ensure that it operates under the standard MMC conditions. Since the scalars do not correlate well with velocity components, we introduce the mixture-fraction-like reference and conditioning variable $\xi_{0}=\eta_{0}$, which append the list of the variables $\eta_{j}=\xi_{j}=u_{j}$, and require that mixing is local with respect to all of the conditioning variables $\left\langle S\left[Y^{(i)}\right] \mid \eta_{0}, \mathbf{u}, \mathbf{x}\right\rangle=0$. Thus, the operator $S$ is now modified

$$
S\left[Y^{(i)}\right]=\frac{\left\langle Y^{(i)} \mid \eta_{0}, \mathbf{u}, \mathbf{x}\right\rangle-Y^{(i)}}{\tau_{S}}
$$

but the coefficients of the model $A_{j}^{\circ}$ (where $j=1,2,3$ ) and $C_{0} \bar{\varepsilon} / 2$ remain the same. The system (51)-(53) is appended by equation $d \xi_{0}=A_{0}^{\circ} d t+b_{00} d w_{0}$ and the choice of $b_{00}^{2}=2 B_{00}$ and $A_{0}^{\circ}$ is subject to the usual constraints for mixture fraction-like reference variables. The equation for $\bar{Z} \equiv\left\langle Z \mid \eta_{0}, \mathbf{u}, \mathbf{x}\right\rangle$ takes the form

$\frac{\partial \bar{Z}}{\partial t}+\mathbf{u} \cdot \nabla \bar{Z}+A_{j} \frac{\partial \bar{Z}}{\partial u_{j}}-\frac{C_{0} \bar{\varepsilon}}{2} \frac{\partial^{2} \bar{Z}}{\partial u_{j} \partial u_{j}}+A_{0} \frac{\partial \bar{Z}}{\partial \eta_{0}}-B_{00} \frac{\partial^{2} \bar{Z}}{\partial \eta_{0}^{2}}=\left\langle S[Z] \mid \eta_{0}, \mathbf{u}, \mathbf{x}\right\rangle=0$

where

$$
A_{0}^{\circ} \equiv A_{0}+\frac{2}{P_{\xi}} \frac{\partial B_{00}\langle\rho\rangle P_{\xi}}{\partial \xi_{0}}
$$

and $P_{\xi}=P\left(\xi_{0}, \mathbf{u}, \mathbf{x} ; t\right)$ is the reference $\mathrm{PDF}$.

The characteristics of the velocity-conditioned scalar $Z$

$$
\bar{Z}_{u}=\langle Z \mid \mathbf{u}, \mathbf{x}\rangle=\int \bar{Z} P\left(\eta_{0} \mid \mathbf{u}, \mathbf{x}\right) d \eta_{0}
$$

remain the same for both of the models. Indeed since both $\bar{Z}_{u}$ and $\bar{Z}$ are not affected by the operator $S$, which is not present in equations (55) and (58), we can put $S=0$ without altering $\bar{Z}_{u}$ and $\bar{Z}$. One can see that, if $S=0$, the appended equation does not have any affect on the rest of the model. Hence, the major transport of the scalar $Z$ in physical space is the same for both of the models. There are however some differences. The additional conditioning variable $\eta_{0}$ allows for reduction of the conditional fluctuations $Z-\bar{Z}$ in the extended model compared with the conditional fluctuations $Z-\bar{Z}_{u}$ present in the model prior to the modification due expectation that the mixture fractionlike reference variable $\eta_{0}$ correlates well with the mixture fraction $Z$. This means the $S$ is better localised and the extended model operates in agreement with MMC principles so that the minor dissipation time is not strictly linked to the overall dissipation rate. As it is discussed in the previous section, these 
goals are easier to achieve when the mixture-fraction-like reference variable $\eta_{0}$ follows the Lagrangian characteristics of the real mixture fraction.

The extended model possesses one significant disadvantage - the computational cost. The large number of conditioning variables needs many particles for accurate evaluation of the conditional expectations. In the framework of the generalised MMC, the computational cost can be reduced by excluding the reference variables $\xi_{j}=u_{j}(j=1,2,3)$ from the list of conditioning variables that are now represented by a single variable, $\eta_{0}=\xi_{0}$. In this reduced version of the model

$$
S\left[Y^{(i)}\right]=\frac{\left\langle Y^{(i)} \mid \eta_{0}, \mathbf{x}\right\rangle-Y^{(i)}}{\tau_{S}}
$$

so that $\left\langle S[Z] \mid \eta_{0}, \mathbf{x}\right\rangle=0$ but $\langle S[Z] \mid \mathbf{u}, \mathbf{x}\rangle \neq 0$. This mixing operator $S$ affects the turbulent transport in any direction that does not coincide with the direction of mixture fraction transport. Note that the standard MMC equation obtained from (58) by omitting the derivatives $\partial / \partial u_{j}$ is, generally, not valid for the reduced model due to cross-correlations involving $u_{j}$ and $Z$. This may be an acceptable price to be paid for computational efficiency in many non-premixed flames where transport of all species is more or less akin that of the mixture fractions. Let us examine whether transport of the mixture fraction in the reduced model is consistent with the transport of the mixture fraction simulated by the extended model. If $A_{0}=0$, the extended model corresponds to equation (58) and that equation allows for solution $\bar{Z}=\eta_{0}$. As it is demonstrated in the previous paragraph, any solution of (58) generates $\langle Z \mid \mathbf{u}, \mathbf{x}\rangle$ that is consistent with the original model and equation (55). Equations (58) can still be used to characterise $\left\langle Z \mid \eta_{0}, \mathbf{u}, \mathbf{x}\right\rangle$ of the reduced model but, generally, $\left\langle S[Z] \mid \eta_{0}, \mathbf{u}, \mathbf{x}\right\rangle \neq 0$ since the reduced model demands only that $\left\langle S[Z] \mid \eta_{0}, \mathbf{x}\right\rangle=0$. However, one can see that in this case $\left\langle S[Z] \mid \eta_{0}, \mathbf{u}, \mathbf{x}\right\rangle=\left\langle\left(\left\langle Z \mid \eta_{0}, \mathbf{x}\right\rangle-Z\right) \mid \eta_{0}, \mathbf{u}, \mathbf{x}\right\rangle / \tau_{S}=0$ for any $Z$ satisfying $\left\langle Z \mid \eta_{0}, \mathbf{u}, \mathbf{x}\right\rangle=\eta_{0}$. Hence $\bar{Z}=\eta_{0}$ remains a valid solution for the reduced model.

Thus, if $A_{0}=0$, the mixture fraction transport is the same for reduced and extended models, although transport in any other direction would be different in these models. The condition $A_{0}=0$ means that the conditioning variable $\eta_{0}$ simulates the mixture fraction. This is quite difficult to achieve with a reasonably simple choice of the coefficients $A_{0}$ and $B_{00}$ due to the fact the conventional mixture fraction values are bounded by 0 and 1 . Thus, in practical modelling, the transport properties of the reduced and extended models would be different even for the mixture fraction. However, if $\eta_{0}$ is reasonably close to the mixture fraction, the transport properties can be expected to be quite similar. The present section demonstrates how the models that comply with both the MMC requirements and with the transport properties of the wellexplored methodology of Lagrangian velocity simulations can be constructed. 


\section{Discussion and Conclusions}

The 2nd order CMC equations with scalar (mixture fraction) conditioning involve conditional generation term that is specific to the conditional variance equations and does not appear in the equation for unconditional scalar variance but can be dominant under typical conditions. The analysis of this term is conducted for locally homogeneous isotropic turbulence on the basis of the theory of the inertial interval for an inert or slowly reacting scalars. The obtained estimates for the generation term involve the characteristic generation time $\tau_{N}$ that is shown to be related to the characteristic time of Lagrangian correlation of the scalar dissipation. Strong and stable chemical reactions are likely to reduce $\tau_{N}$. The rate of generation of conditional fluctuations is also determined for the major mixing models and it is shown that this rate exhibits a non-trivial dependence on the model parameters that may include the duration of the time step. An accurate implementation of a mixing model should involve a proper selection of the model parameters that allows to set the conditional generation rate at the desired level. Matching the expected physical conditional generation rate is even more important in MMC modelling. In MMC, one of the model parameters - the minor mixing time scale - should be explicitly selected to produce the required level of the conditional fluctuations. The conditional generation rate in the simplest version of MMC with a single mixture fraction-like reference variable and the minor dissipation operator represented by Curl's mixing model is analysed and shown to be consistent with the physical expectations for the rate.

While the first part of this work is mainly concerned with the mixture fractiontype conditioning, a more general understanding of MMC modelling is introduced in the second part. The generalised MMC involves several conditioning variables $\eta_{1}, \ldots, \eta_{n_{c}}$ that are used to emulate selected Lagrangian properties of turbulence (such as the scalar dissipation and velocities). The conditioning variables depend on the set of the reference variables $\xi_{1}, \ldots, \xi_{n_{r}}$ that do not necessarily coincide with the conditioning variables. The reference variables are used to assist the emulation process. Another set of variables $Z^{(i)}$ simulates the reacting scalars. These variables are affected by the reaction source terms and by the surrogate mixing operator utilising the conventional mixing models. The MMC methodology allows to enforce the desired conditional characteristics $\left\langle Z^{(i)} \mid \boldsymbol{\eta}\right\rangle$ through adequate emulation of Lagrangian properties by $\boldsymbol{\eta}(t)$. The influence of the surrogate mixing is restricted to the local treatment of the fluctuations with respect to $\left\langle Z^{(i)} \mid \boldsymbol{\eta}\right\rangle$ (the minor fluctuations) and, to a certain extent, this negates the deficiencies of surrogate mixing.

The last section of the present paper demonstrates how consistent MMC models with various types of conditioning can be constructed. The Lagrangian properties of the scalar dissipation with a log-normal distribution and required 
correlation function are emulated by a proper choice of the reference variables that, effectively, represents the turbulent cascade. The spatial turbulent transport of scalars can, presumably, be simulated by IECM model. The model is converted into a proper MMC model in a way that preserves the transport characteristics. The emulation of some selected properties of turbulence (the mixture fraction and the velocities but not the dissipation) can be replaced by LES of these values. Combining MMC and LES allows to evaluate the large-scale transport by LES while the small-scale dissipation characteristics are emulated by MMC.

It seems that the future of turbulent combustion modelling is not in the use of a particular model but in combining the models in a way that amplifies their strong features and compensates their deficiencies. Ideally, the user should be able to "order" the desired features of the model, although the additional features are likely to come at some additional computational cost. For example, if the user is not satisfied with Lagrangian stochastic simulation of velocities then, presumably, LES can reproduce the Lagrangian trajectories better while the rest of the combustion model does not need to change much. It seems that $\mathrm{MMC}$ is a right methodology for flexible and consistent interactions between the models.

\section{References}

[1] R.W. Bilger. Turbulent flows with nonpremixed reactants. In P.A. Libby and F.A. Williams, editors, Turbulent Reacting Flows. Springer Verlag, 1980.

[2] N. Peters. Turbulent Combustion. Cambridge University Press, 2000.

[3] S.B. Pope. Pdf methods for turbulent reactive flows. Prog. Energy Combust. Sci., 11:119-192, 1985.

[4] C. Dopazo. Recent developments in Pdf methods. In P. A. Libby and F. A. Williams, editors, Turbulent Reacting Flows, pages 375-474. Academic Press, 1994.

[5] A.Y. Klimenko and R.W. Bilger. Conditional moment closure for turbulent combustion. Prog. Energy Combust. Sci., 25:595-687, 1999.

[6] S. B. Pope. Accessed compositions in turbulent reactive flows. Flow, Turbulence and Combustion, 72:219-243, 2004.

[7] A.Y. Klimenko and S.B. Pope. A model for turbulent reactive flows based on multiple mapping conditioning. Physics of Fluids, 15:1907-1925, 2003.

[8] A. Y. Klimenko. Matching the conditional variance as a criterion for selecting parameters in the simplest multiple mapping conditioning models. Phys. Fluids, 16:4754-4757, 2004. 
[9] H. Chen, S. Chen, and R. H. Kraichnan. Probability distribution of a stochastically advected scalar field. Phys. Rev. Lett., 63:2657-2660, 1989.

[10] S. B. Pope. Mapping closures for turbulent mixing and reaction. Theor. Comput. Fluid Dyn., 2:255-270, 1991.

[11] F. Gao. An analytic solution for the scalar probability density function in homogeneous turbulence. Phys. Fluids A, 3:511-513, 1991.

[12] C. M. Cha, G. Kosaly, and H. Pitsch. Modelling extinction and reignition in turbulent nonpremixed combustion using a doubly-conditioned moment closure approach. Phys. Fluids, 13:3824-3834, 2001.

[13] S.H.Kim. On the conditional variance and covariance equations for second-order conditional moment closure. Phys. Fluids, 14:2011-2014, 2002.

[14] A.Y. Klimenko. Conditional moment closure and fluctuations of scalar dissipation. Fluid Dynamics, 28:630-637, 1993.

[15] N. Swaminathan and R. W. Bilger. Study of the conditional covarience and variance equations for the second order conditional moment closure. Phys. Fluids, 11:2679-2695, 1999.

[16] C. Dopazo. Probability density function approach for a turbulent axisymmetric heated jet centreline evolution. Phys. Fluids, 18:397-410, 1975.

[17] J. Janicka, W. Kolbe, and W. Kollmann. Closure of the transport equation for the probability density function of scalar fields. J. Non-equilib. Thermodyn, $4(1): 47-66,1979$.

[18] R.P. Lindstedt, S.A. Louloudi, J.J. Driscoll, and V. Sick. Finite rate chemistry effects in turbulent reacting flows. Flow, Turbulence and Combustion, 72:407426, 2004.

[19] S. Subramaniam and S. B. Pope. A mixing model for turbulent reactive flows based on Euclidean minimum spanning trees. Combust. Flame, 115:487-514, 1998.

[20] R. O. Fox. On velocity-conditioned scalar mixing in homogeneous turbulence. Physics of Fluids, 8(10):2678-2691, oct 1996.

[21] K. R. Sreenivasan. Possible effects of small-scale intermittency in turbulent reacting flows. Flow, Turbulence and Combustion, 72:115-131, 2004.

[22] A. Y. Klimenko. Mmc modelling and fluctuations of the scalar dissipation. In Aust. Comb. Symposium, 2003.

[23] S. B. Pope. On the relationship between stochastic Lagrangian models of turbulence and second-moment closures. Phys. Fluids, 6:973-985, 1994.

[24] B. Sawford. Micro-mixing modelling of scalar fluctuations for plumes in homogeneous turbulence. Flow, Turbulence and Combustion, 72:133-160, 2004. 
[25] A. Y. Klimenko. Conditional methods in application for Lagrangian modelling. Physics of Fluids, 10:922-927, 1998.

\section{Nomenclature}

$Y$ reactive scalars

$y=Y-\langle Y\rangle$

$Z$ inert scalar or mixture fraction

$z=Z-\langle Z\rangle$

$Q$ conditional average $=\langle Y \mid Z\rangle$

$\Theta$ conditional variance $=\left\langle(Y-Q)^{2} \mid Z\right\rangle$

$f$ functional depedence $Y=f(Z)$

$\Psi$ generation and dissipation terms in 2nd order CMC

u velocity

$\mathbf{x}$ physical coordinate

$N$ scalar dissipation

$W$ chemical source term

$P$ probability density function

$\rho$ density

$S$ mixing operator

$D$ molecular diffusion coefficient

$t$ time

$\tau$ time scale

$K$ correlation of the scalar dissipation

$\xi$ MMC reference scalars

$\eta$ MMC conditioning scalars 
$A$ drift coefficient (in the reference space)

$B$ diffusion coefficient (in the reference space)

$w$ standard Wiener process 